\title{
Kaplan classes of a certain family of functions
}

\begin{abstract}
We give the complete characterization of members of Kaplan classes of products of power functions with all zeros symmetrically distributed in $\mathbb{T}:=\{z \in \mathbb{C}:|z|=1\}$ and weakly monotonic sequence of powers. In this way we extend Sheil-Small's theorem. We apply the obtained result to study univalence of antiderivative of these products of power functions.
\end{abstract}

Introduction. Let $\mathcal{H}_{d}$ be the class of all analytic functions $f: \mathbb{D} \rightarrow \mathbb{C}$ normalized by $f(0)=1$ and such that $f \neq 0$ in $\mathbb{D}:=\{z \in \mathbb{C}:|z|<1\}$. Let $\mathcal{S}$ be the class of all analytic functions $f: \mathbb{D} \rightarrow \mathbb{C}$ normalized by $f(0)=f^{\prime}(0)-1=0$ which are univalent and $\mathcal{C}$ be the class of functions in $\mathcal{S}$ that are close-to-convex. For $\alpha, \beta \geq 0$ the Kaplan class $K(\alpha, \beta)$ is the set of all functions $f \in \mathcal{H}_{d}$ satisfying one of the two equivalent conditions:

$$
\begin{aligned}
& \arg f\left(r \mathrm{e}^{\mathrm{i} \theta_{2}}\right)-\arg f\left(r \mathrm{e}^{\mathrm{i} \theta_{1}}\right) \leq \beta \pi-\frac{1}{2}(\alpha-\beta)\left(\theta_{1}-\theta_{2}\right), \\
& -\alpha \pi-\frac{1}{2}(\alpha-\beta)\left(\theta_{1}-\theta_{2}\right) \leq \arg f\left(r \mathrm{e}^{\mathrm{i} \theta_{2}}\right)-\arg f\left(r \mathrm{e}^{\mathrm{i} \theta_{1}}\right) .
\end{aligned}
$$

for $0<r<1$ and $\theta_{1}<\theta_{2}<\theta_{1}+2 \pi$ (see [6, pp. 32-33]).

Let $\mathbb{N}_{j}:=\mathbb{N} \cap[1 ; j]$ for $j \in \mathbb{N}$ and $\mathbb{R}^{+}:=(0 ;+\infty)$. Fix $n \in \mathbb{N}$ and a weakly monotonic sequence $m: \mathbb{N}_{n} \rightarrow \mathbb{R}^{+}$. Define the functions

$$
\mathbb{D} \ni z \mapsto f_{k}(z):=1-z \mathrm{e}^{-\mathrm{i} \frac{2 \pi(k-1)}{n}} \text { for } k \in \mathbb{N}_{n}
$$

2010 Mathematics Subject Classification. Primary: 30C15; secondary: 30C45, 30C55.

Key words and phrases. Kaplan classes, univalence, close-to-convex functions, critical points. 
and

$$
\mathbb{D} \ni z \mapsto P_{n}(z ; m):=\prod_{k=1}^{n} f_{k}^{m_{k}}(z) .
$$

We denote the class of all such functions $P_{n}(\cdot ; m)$ by $\mathcal{P}_{n}$. Let us notice that the function $P_{n}(\cdot ; m)$ is a product of power functions with all zeros symmetrically distributed in $\mathbb{T}$. In particular if $m_{k} \in \mathbb{N}$ for all $k \in \mathbb{N}_{n}$, then $P_{n}(\cdot ; m)$ is a polynomial of degree $\sum_{k=1}^{n} m_{k}$ with all zeros symmetrically distributed in $\mathbb{T}$. The functions of the form $\mathbb{D} \ni z \mapsto 1-z \mathrm{e}^{-\mathrm{i} t}$ for $t \in[0 ; 2 \pi)$ play the central role in the univalent functions theory. Due to the result of Royster [5] they are used for example as an extremal functions in many articles (see $[1,4])$.

The Kaplan classes were used as the universal tool for establishing many important subclasses of $\mathcal{S}$ (see [6, p. 47]). Complete membership study even for the simplest functions from $\mathcal{H}_{d}$ was not carried out. For a given function it can be difficult to check if it belongs to any Kaplan class. We deduce from $\left[2\right.$, Theorem 1.1] that $f_{k} \in K(1,0)$ for any $k \in \mathbb{N}_{n}$. Moreover, Sheil-Small proved the following theorem (see [7, p. 248]).

Theorem A (Sheil-Small). For any polynomial $Q \in \mathcal{H}_{d}$ of the degree $n \in \mathbb{N} \backslash\{1\}$ with all zeros in $\mathbb{T}$, if $\lambda$ is the minimal arclength between two consecutive zeros of $Q$, then $Q \in K(1,2 \pi / \lambda-n+1)$.

Theorem A can also be deduced from [3], where Jahangiri obtained a certain gap condition for polynomials with all zeros in $\mathbb{T}$. In [2], we extended the Jahangiri's result for all $\alpha, \beta \geq 0$ and effectively determined complete membership to Kaplan classes of polynomials with all zeros in $\mathbb{T}$. In this article, we extend the above results by describing complete membership to Kaplan classes of functions from the class $\mathcal{P}_{n}$ for all $n \in \mathbb{N}$. To this end we recall some properties of Kaplan classes (see [7, p. 245]).

Lemma B. For all $\alpha_{1}, \alpha_{2}, \beta_{1}, \beta_{2} \geq 0$ and $t>0$ the following conditions hold:

$$
\begin{aligned}
& f \in K\left(\alpha_{1}, \beta_{1}\right) \text { and } g \in K\left(\alpha_{2}, \beta_{2}\right) \Rightarrow f g \in K\left(\alpha_{1}+\alpha_{2}, \beta_{1}+\beta_{2}\right), \\
& f \in K\left(\alpha_{1}, \beta_{1}\right) \Rightarrow f^{0} \in K(0,0), \\
& f \in K\left(\alpha_{1}, \beta_{1}\right) \Longleftrightarrow f^{t} \in K\left(t \alpha_{1}, t \beta_{1}\right), \\
& f \in K\left(\alpha_{1}, \beta_{1}\right) \Longleftrightarrow f^{-1} \in K\left(\beta_{1}, \alpha_{1}\right) .
\end{aligned}
$$


1. Main theorems. Assume that $m_{0}:=0$. For all $j \in \mathbb{N}$ and $k \in \mathbb{N}_{n}$ we define

$$
\begin{aligned}
& t_{j}:= \frac{2 \pi(j-1)}{n}, \quad s:=\sum_{l=1}^{n} m_{l}, \\
& a_{k}:=-\frac{n-k}{k}, \quad b_{k}:=-s+\frac{n}{k} \sum_{l=n-k}^{n} m_{l}, \\
& x_{k}:=\sum_{l=n-k}^{n} m_{l}-k m_{n-k}, \quad y_{k}:=(n-k) m_{n-k}-\sum_{l=1}^{n-k-1} m_{l}, \\
& \quad \Pi_{0}:=\left\{(x, y) \in \mathbb{R}^{2}: x \geq m_{n}\right\}, \\
& \quad \Pi_{k}:=\left\{(x, y) \in \mathbb{R}^{2}: y \geq a_{k} x+b_{k}\right\}, \\
& \Pi_{0}^{\prime}:=\left\{(x, y) \in \mathbb{R}^{2}: 0 \leq x<m_{n}\right\}, \\
& \Pi_{k}^{\prime}:=\left\{(x, y) \in \mathbb{R}^{2}: 0 \leq x, 0 \leq y<a_{k} x+b_{k}\right\}, \\
& \quad \Pi_{l}^{n}:=\bigcap_{l=0}^{n} \Pi_{l} .
\end{aligned}
$$

Now we give the complete characterization of membership of $P_{n}(\cdot ; m)$ to Kaplan classes.

Theorem 1.1. If $m: \mathbb{N}_{n} \rightarrow \mathbb{R}^{+}$is weakly monotonic, then for all $\alpha, \beta \geq 0$, $P_{n}(\cdot ; m) \in K(\alpha, \beta)$ if and only if $(\alpha, \beta) \in \Pi$.

Proof. Without loss of generality we assume that $m$ is a nondecreasing sequence. Since $\prod_{k=1}^{n} f_{k}(z)=1-z^{n}$ and $1-z^{n}$ has positive real part in $\mathbb{D}$, we have

$$
\prod_{k=1}^{n} f_{k} \in K(1,1) .
$$

First we prove that $P_{n}(\cdot ; m) \in K\left(x_{k}, y_{k}\right)$ for $k \in \mathbb{N}_{n}$. Fix $k \in \mathbb{N}_{n}$. Therefore,

$$
\begin{aligned}
P_{n}(\cdot ; m) & =\prod_{l=1}^{n} f_{l}^{m_{n-k}} \prod_{l=1}^{n} f_{l}^{m_{l}-m_{n-k}} \\
& =\prod_{l=1}^{n} f_{l}^{m_{n-k}} \prod_{l=1}^{n-k-1} f_{l}^{m_{l}-m_{n-k}} \prod_{l=n-k+1}^{n} f_{l}^{m_{l}-m_{n-k}} \\
& =\prod_{l=1}^{n} f_{l}^{m_{n-k}} \prod_{l=1}^{n-k-1}\left(\frac{1}{f_{l}}\right)^{m_{n-k}-m_{l}} \prod_{l=n-k+1}^{n} f_{l}^{m_{l}-m_{n-k}} .
\end{aligned}
$$


By (1.1) and Lemma B, we get

$$
\begin{aligned}
\prod_{l=1}^{n} f_{l}^{m_{n-k}} & \in K\left(m_{n-k}, m_{n-k}\right), \\
\left(1 / f_{l}\right)^{m_{n-k}-m_{l}} & \in K\left(0, m_{n-k}-m_{l}\right) \text { for } l \in \mathbb{N}_{n-k-1}
\end{aligned}
$$

and

$$
f_{l}^{m_{l}-m_{n-k}} \in K\left(m_{l}-m_{n-k}, 0\right) \text { for } l \in \mathbb{N}_{n} \backslash \mathbb{N}_{n-k} .
$$

Then

$$
P_{n}(\cdot ; m) \in K\left(m_{n-k}+\sum_{l=n-k+1}^{n}\left(m_{l}-m_{n-k}\right), m_{n-k}+\sum_{l=1}^{n-k-1}\left(m_{n-k}-m_{l}\right)\right)
$$

and as a consequence

$$
P_{n}(\cdot ; m) \in K\left(x_{k}, y_{k}\right) .
$$

By Lemma B, we obtain $f \in \Pi$.

Now we prove the second part of the theorem. Fix $k \in \mathbb{N}_{n-1}$. Consider the left side of inequality $(0.1)$ with $\mathbb{N} \ni j \mapsto \theta_{1}(j):=-2 \pi / n+1 / j$, $\mathbb{N} \ni j \mapsto \theta_{2}(j):=2 \pi-2 \pi(k+1) / n-1 / j$ and $\mathbb{N} \ni j \mapsto r_{j}:=1-1 / j^{2}$. Therefore,

$$
\begin{aligned}
& \arg \left(P_{n}\left(r_{j} \mathrm{e}^{\mathrm{i} \theta_{2}} ; m\right)\right)-\arg \left(P_{n}\left(r_{j} \mathrm{e}^{\mathrm{i} \theta_{1}} ; m\right)\right) \\
& =\sum_{l=1}^{n} m_{l}\left(\arctan \left(\frac{-r_{j} \sin \left(\theta_{2}(j)-\frac{2 \pi}{n}(l-1)\right)}{1-r_{j} \cos \left(\theta_{2}(j)-\frac{2 \pi}{n}(l-1)\right)}\right)\right. \\
& \left.-\arctan \left(\frac{-r_{j} \sin \left(\theta_{1}(j)-\frac{2 \pi}{n}(l-1)\right)}{1-r_{j} \cos \left(\theta_{1}(j)-\frac{2 \pi}{n}(l-1)\right)}\right)\right) \\
& =\sum_{l=1}^{n} m_{l}\left(\arctan \left(\frac{r_{j} \sin \left(\frac{2 \pi}{n}(k+l)+\frac{1}{j}\right)}{1-r_{j} \cos \left(\frac{2 \pi}{n}(k+l)+\frac{1}{j}\right)}\right)\right. \\
& =\sum_{l=1}^{n-k-1} m_{l}\left(\arctan \left(\frac{r_{j} \sin \left(\frac{2 \pi l}{n}-\frac{1}{j}\right)}{1-r_{j} \cos \left(\frac{2 \pi l}{n}-\frac{1}{j}\right)}\right)\right) \\
& \left(\frac{r_{j} \sin \left(\frac{2 \pi}{n}(k+l)+\frac{1}{j}\right)}{1-r_{j} \cos \left(\frac{2 \pi}{n}(k+l)+\frac{1}{j}\right)}\right) \\
& \left.-\arctan \left(\frac{r_{j} \sin \left(\frac{2 \pi l}{n}-\frac{1}{j}\right)}{1-r_{j} \cos \left(\frac{2 \pi l}{n}-\frac{1}{j}\right)}\right)\right)
\end{aligned}
$$




$$
\begin{aligned}
& +\sum_{l=n-k+1}^{n-1} m_{l}\left(\arctan \left(\frac{r_{j} \sin \left(\frac{2 \pi}{n}(k+l)+\frac{1}{j}\right)}{1-r_{j} \cos \left(\frac{2 \pi}{n}(k+l)+\frac{1}{j}\right)}\right)\right. \\
& \left.-\arctan \left(\frac{r_{j} \sin \left(\frac{2 \pi l}{n}-\frac{1}{j}\right)}{1-r_{j} \cos \left(\frac{2 \pi l}{n}-\frac{1}{j}\right)}\right)\right) \\
& +m_{n-k}\left(\arctan \left(\frac{\left(1-\frac{1}{j^{2}}\right) \sin \left(\frac{1}{j}\right)}{1-\left(1-\frac{1}{j^{2}}\right) \cos \left(\frac{1}{j}\right)}\right)\right. \\
& +m_{n}\left(\arctan \left(\frac{r_{j} \sin \left(\frac{2 \pi k}{n}+\frac{1}{j}\right)}{1-r_{j} \cos \left(\frac{2 \pi k}{n}+\frac{1}{j}\right)}\right)\right) \\
& \left.+\frac{r_{j} \sin \left(\frac{2 \pi k}{n}+\frac{1}{j}\right)}{1-r_{j} \cos \left(\frac{2 \pi k}{n}+\frac{1}{j}\right)}\right) \\
& \left.+\arctan \left(\frac{\left(1-\frac{1}{j^{2}}\right) \sin \left(\frac{1}{j}\right)}{1-\left(1-\frac{1}{j^{2}}\right) \cos \left(\frac{1}{j}\right)}\right)\right)
\end{aligned}
$$

and as a consequence

$$
\begin{aligned}
& \lim _{j \rightarrow+\infty}\left(\arg \left(P_{n}\left(r_{j} \mathrm{e}^{\mathrm{i} \theta_{2}} ; m\right)\right)-\arg \left(P_{n}\left(r_{j} \mathrm{e}^{\mathrm{i} \theta_{1}} ; m\right)\right)\right) \\
& =\sum_{l=1}^{n-k-1} m_{l}\left(\arctan \left(\frac{\sin \left(\frac{2 \pi}{n}(k+l)\right)}{1-\cos \left(\frac{2 \pi}{n}(k+l)\right)}\right)-\arctan \left(\frac{\sin \left(\frac{2 \pi l}{n}\right)}{1-\cos \left(\frac{2 \pi l}{n}\right)}\right)\right) \\
& +\sum_{l=n-k+1}^{n-1} m_{l}\left(\arctan \left(\frac{\sin \left(\frac{2 \pi}{n}(k+l)\right)}{1-\cos \left(\frac{2 \pi}{n}(k+l)\right)}\right)-\arctan \left(\frac{\sin \left(\frac{2 \pi l}{n}\right)}{1-\cos \left(\frac{2 \pi l}{n}\right)}\right)\right) \\
& +m_{n-k}\left(\frac{\pi}{2}+\arctan \left(\frac{\sin \left(\frac{2 \pi k}{n}\right)}{1-\cos \left(\frac{2 \pi k}{n}\right)}\right)\right) \\
& +m_{n}\left(\arctan \left(\frac{\sin \left(\frac{2 \pi k}{n}\right)}{1-\cos \left(\frac{2 \pi k}{n}\right)}\right)+\frac{\pi}{2}\right) .
\end{aligned}
$$

By the trigonometric identity:

$$
\frac{\sin x}{1-\cos x}=\tan \left(\frac{\pi}{2}-\frac{x}{2}\right) \quad \text { for } \quad x \in \mathbb{R} \backslash \bigcup_{j \in \mathbb{Z}}\{2 j \pi\}
$$




$$
\begin{aligned}
& \text { we get } \\
& \begin{array}{l}
\lim _{j \rightarrow+\infty}\left(\arg \left(P_{n}\left(r_{j} \mathrm{e}^{\mathrm{i} \theta_{2}} ; m\right)\right)-\arg \left(P_{n}\left(r_{j} \mathrm{e}^{\mathrm{i} \theta_{1}} ; m\right)\right)\right) \\
=\sum_{l=1}^{n-k-1} m_{l}\left(\arctan \left(\tan \left(\frac{\pi}{2}-\frac{\pi}{n}(k+l)\right)\right)-\arctan \left(\tan \left(\frac{\pi}{2}-\frac{\pi l}{n}\right)\right)\right) \\
\quad+\sum_{l=n-k+1}^{n-1} m_{l}\left(\arctan \left(\tan \left(\frac{\pi}{2}-\frac{\pi}{n}(k+l)\right)\right)-\arctan \left(\tan \left(\frac{\pi}{2}-\frac{\pi l}{n}\right)\right)\right) \\
\quad+m_{n-k}\left(\frac{\pi}{2}+\arctan \left(\tan \left(\frac{\pi}{2}-\frac{\pi k}{n}\right)\right)\right) \\
\quad+m_{n}\left(\arctan \left(\tan \left(\frac{\pi}{2}-\frac{\pi k}{n}\right)\right)+\frac{\pi}{2}\right) .
\end{array}
\end{aligned}
$$

Since

$$
\begin{aligned}
& \frac{\pi}{2}-\frac{\pi}{n}(k+l) \in\left(-\frac{\pi}{2} ; \frac{\pi}{2}\right) \text { for } l \in \mathbb{N}_{n-k-1}, \\
& \frac{\pi}{2}-\frac{\pi}{n}(k+l) \in\left(-\frac{3 \pi}{2} ;-\frac{\pi}{2}\right) \text { for } l \in \mathbb{N}_{n-1} \backslash \mathbb{N}_{n-k}
\end{aligned}
$$

and

$$
\frac{\pi}{2}-\frac{\pi l}{n} \in\left(-\frac{\pi}{2} ; \frac{\pi}{2}\right) \text { for } l \in \mathbb{N}_{n-1}
$$

we have

$$
\begin{aligned}
& \lim _{j \rightarrow+\infty}\left(\arg \left(P_{n}\left(r_{j} \mathrm{e}^{\mathrm{i} \theta_{2}} ; m\right)\right)-\arg \left(P_{n}\left(r_{j} \mathrm{e}^{\mathrm{i} \theta_{1}} ; m\right)\right)\right) \\
= & \sum_{l=1}^{n-k-1} m_{l}\left(\frac{\pi}{2}-\frac{\pi}{n}(k+l)-\frac{\pi}{2}+\frac{\pi l}{n}\right) \\
& +\sum_{l=n-k+1}^{n-1} m_{l}\left(\frac{3 \pi}{2}-\frac{\pi}{n}(k+l)-\frac{\pi}{2}+\frac{\pi l}{n}\right) \\
& +m_{n-k}\left(\frac{\pi}{2}+\frac{\pi}{2}-\frac{\pi k}{n}\right)+m_{n}\left(\frac{\pi}{2}-\frac{\pi k}{n}+\frac{\pi}{2}\right) \\
= & -\frac{\pi k s}{n}+\pi \sum_{l=n-k}^{n} m_{l} .
\end{aligned}
$$

On the other hand,

$$
\begin{aligned}
\lim _{j \rightarrow+\infty}\left(\beta \pi+\frac{1}{2}(\alpha-\beta)\left(\theta_{2}(j)-\theta_{1}(j)\right)\right) & =\beta \pi+(\alpha-\beta)\left(\pi-\frac{\pi k}{n}\right) \\
& =\beta \pi \frac{k}{n}+\alpha \pi \frac{n-k}{n},
\end{aligned}
$$


from which we deduce that inequality (0.1) does not hold for

$$
\beta<-\frac{n-k}{k} \alpha-s+\frac{n}{k} \sum_{l=n-k}^{n} m_{l}
$$

and as a consequence $P_{n}(\cdot ; m) \notin K(\alpha, \beta)$ for $(\alpha, \beta) \in \Pi_{k}^{\prime}$. Hence

$$
P_{n}(\cdot ; m) \notin \bigcup_{k=1}^{n-1} \Pi_{k}^{\prime} .
$$

Now we prove that $P_{n}(\cdot ; m) \notin \Pi_{0}^{\prime}$. Consider the right side of inequality $(0.2)$ with $\mathbb{N} \ni j \mapsto \theta_{1}(j):=2 \pi(n-1) / n+1 / j, \mathbb{N} \ni j \mapsto \theta_{2}(j):=2 \pi(n-1) / n-1 / j$ and $\mathbb{N} \ni j \mapsto r_{j}:=1-1 / j^{2}$. Therefore,

$$
\begin{aligned}
& \arg \left(P_{n}\left(r_{j} \mathrm{e}^{\mathrm{i} \theta_{2}} ; m\right)\right)-\arg \left(P_{n}\left(r_{j} \mathrm{e}^{\mathrm{i} \theta_{1}} ; m\right)\right) \\
& =-2 m_{n} \arctan \left(\frac{\left(1-\frac{1}{j^{2}}\right) \sin \left(\frac{1}{j}\right)}{1-\left(1-\frac{1}{j^{2}}\right) \cos \left(\frac{1}{j}\right)}\right) \\
& +\sum_{l=1}^{n-1} m_{l}\left(\arctan \left(\frac{-r_{j} \sin \left(\frac{2 \pi l}{n}-\frac{1}{j}\right)}{1-r_{j} \cos \left(\frac{2 \pi l}{n}-\frac{1}{j}\right)}\right)\right. \\
& \left.-\arctan \left(\frac{-r_{j} \sin \left(\frac{2 \pi l}{n}+\frac{1}{j}\right)}{1-r_{j} \cos \left(\frac{2 \pi l}{n}+\frac{1}{j}\right)}\right)\right)
\end{aligned}
$$

and as a consequence

$$
\lim _{j \rightarrow+\infty}\left(\arg \left(P_{n}\left(r_{j} \mathrm{e}^{\mathrm{i} \theta_{2}} ; m\right)\right)-\arg \left(P_{n}\left(r_{j} \mathrm{e}^{\mathrm{i} \theta_{1}} ; m\right)\right)\right)=-m_{n} \pi .
$$

On the other hand, we have

$$
\lim _{j \rightarrow+\infty}\left(-\alpha \pi+\frac{1}{2}(\alpha-\beta)\left(\theta_{2}(j)-\theta_{1}(j)\right)\right)=-\alpha \pi,
$$

from which we deduce that inequality (0.2) does not hold for $\alpha<m_{n}$ and as a consequence $P_{n}(\cdot ; m) \notin K(\alpha, \beta)$ for $(\alpha, \beta) \in \Pi_{0}^{\prime}$. From this and (1.3) we obtain

$$
P_{n}(\cdot ; m) \notin \bigcup_{k=0}^{n} \Pi_{k}^{\prime} .
$$

By Theorem A, if $m_{k}=1$ for all $k \in \mathbb{N}_{n}$, then $P_{n}(\cdot ; m) \in K(1,1)$. Theorem 1.1 is an extension of Theorem A for functions from the class $\mathcal{P}_{n}$. Moreover, in the first part of the proof of Theorem 1.1 we obtain nontrivial, interesting factorization of $P_{n}(\cdot ; m)$ (cf. [7, p. 246]). 
Remark 1.2. Let us notice that for a nondecreasing sequence $m: \mathbb{N}_{n} \rightarrow \mathbb{R}^{+}$ points $\left(x_{k}, y_{k}\right)$ for $k \in \mathbb{N}_{n}$ are all vertices of the set $\Pi$. Analogously we can effectively determine vertices of $\Pi$ if $m$ is nonincreasing.

Let $\varphi_{q}^{\prime}:=\left(P_{n}(\cdot ; m)\right)^{q}$ for any $q \in \mathbb{R}$ such that $\varphi_{q}(0)=0$. The complete characterization of functions $P_{n}(\cdot ; m)$ belonging to Kaplan classes obtained in Theorem 1.1 can be used to study univalence of $\varphi_{q}$.

Theorem 1.3. If $m: \mathbb{N}_{n} \rightarrow \mathbb{R}^{+}$is nondecreasing sequence, then for any $n \in \mathbb{N}, k \in \mathbb{N}_{n-1}$ and $q \geq 0$ the following implications hold:

$$
\begin{gathered}
s \geq n m_{n-1}-2 m_{n} \Longrightarrow\left(\varphi_{q} \in \mathcal{C} \Longleftrightarrow q \in\left[0 ; \frac{1}{m_{n}}\right]\right), \\
s \in\left[(n+2 k) m_{n-k-1}-2 \sum_{l=n-k}^{n} m_{l} ;(n+2 k) m_{n-k}-2 \sum_{l=n-k}^{n} m_{l}\right) \\
\Longrightarrow\left(\varphi_{q} \in \mathcal{C} \Longleftrightarrow q \in\left[0 ; \frac{n+2 k}{n \sum_{l=n-k}^{n} m_{l}-k s}\right]\right) .
\end{gathered}
$$

Proof. Let $m$ be a nondecreasing sequence. Fix $q \geq 0$. First we prove (1.4). If $s \geq n m_{n-1}-2 m_{n}$, then $y_{1} \leq 3 x_{1}$. This and Theorem 1.1 imply that $P_{n}(\cdot ; m) \in K\left(m_{n}, 3 m_{n}\right)$ and for any $\alpha \in\left[0 ; m_{n}\right), P_{n}(\cdot ; m) \notin K(\alpha, 3 \alpha)$. Therefore, $\left(P_{n}(\cdot ; m)\right)^{q} \in K(1,3)$ if and only if $q \in\left[0 ; 1 / m_{n}\right]$.

Now we prove (1.5). Fix $k \in \mathbb{N}_{n-1}$. Assume that

$$
s \in\left[(n+2 k) m_{n-k-1}-2 \sum_{l=n-k}^{n} m_{l} ;(n+2 k) m_{n-k}-2 \sum_{l=n-k}^{n} m_{l}\right) .
$$

Then

$$
\begin{cases}y_{l}>3 x_{l} & \text { for } l \in \mathbb{N}_{k}, \\ y_{l} \leq 3 x_{l} & \text { for } l \in \mathbb{N}_{n} \backslash \mathbb{N}_{k} .\end{cases}
$$

This and Theorem 1.1 imply that

$$
P_{n}(\cdot ; m) \in K\left(\frac{n}{n+2 k} \sum_{l=n-k}^{n} m_{l}-k s, \frac{3 n}{n+2 k} \sum_{l=n-k}^{n} m_{l}-k s\right)
$$

and for any

$$
\alpha \in\left[0 ; \frac{n}{n+2 k} \sum_{l=n-k}^{n} m_{l}-k s\right),
$$

$P_{n}(\cdot ; m) \notin K(\alpha, 3 \alpha)$, which leads to $(1.5)$.

Theorem 1.4. If $m: \mathbb{N}_{n} \rightarrow \mathbb{R}^{+}$is a nondecreasing sequence, then for any $n \in \mathbb{N}, k \in \mathbb{N}_{n-1}$ and $q<0$ the following implications hold:

(1.6) $s \geq \frac{2}{3} m_{n}+n m_{n-1} \Longrightarrow\left(\varphi_{q} \in \mathcal{C} \Longleftrightarrow q \in\left[-\frac{3}{m_{n}} ; 0\right)\right)$, 


$$
\begin{gathered}
s \in\left[\left(n-\frac{2}{3} k\right) m_{n-k-1}+\frac{2}{3} \sum_{l=n-k}^{n} m_{l} ;\left(n-\frac{2}{3} k\right) m_{n-k}+\frac{2}{3} \sum_{l=n-k}^{n} m_{l}\right) \\
\Longrightarrow\left(\varphi_{q} \in \mathcal{C} \Longleftrightarrow q \in\left[\frac{3 n-2 k}{k s-n \sum_{l=n-k}^{n} m_{l}} ; 0\right)\right) .
\end{gathered}
$$

Proof. Let $m$ be a nondecreasing sequence. Fix $q<0$. First we prove (1.6). If $s \geq 2 / 3 m_{n}+n m_{n-1}$, then $3 y_{1} \leq x_{1}$. This and Theorem 1.1 imply that $P_{n}(\cdot ; m) \in K\left(m_{n}, 1 / 3 m_{n}\right)$ and for any $\alpha \in\left[0 ; m_{n}\right), P_{n}(\cdot ; m) \notin K(\alpha, 1 / 3 \alpha)$. Therefore, $\left(P_{n}(\cdot ; m)\right) \in K(1,3)$ if and only if $q \in\left[-3 / m_{n} ; 0\right)$.

Now we prove (1.7). Fix $k \in \mathbb{N}_{n-1}$. Assume that

$$
s \in\left[\left(n-\frac{2}{3} k\right) m_{n-k-1}+\frac{2}{3} \sum_{l=n-k}^{n} m_{l} ;\left(n-\frac{2}{3} k\right) m_{n-k}+\frac{2}{3} \sum_{l=n-k}^{n} m_{l}\right) .
$$

Then

$$
\begin{cases}3 y_{l}>x_{l} & \text { for } l \in \mathbb{N}_{k} \\ 3 y_{l} \leq x_{l} & \text { for } l \in \mathbb{N}_{n} \backslash \mathbb{N}_{k} .\end{cases}
$$

This and Theorem 1.1 imply that

$$
P_{n}(\cdot ; m) \in K\left(\frac{3 n}{3 n-2 k} \sum_{l=n-k}^{n} m_{l}-k s, \frac{n}{3 n-2 k} \sum_{l=n-k}^{n} m_{l}-k s\right)
$$

and for any

$$
\alpha \in\left[0 ; \frac{n}{3 n-2 k} \sum_{l=n-k}^{n} m_{l}-k s\right),
$$

$P_{n}(\cdot ; m) \notin K(3 \alpha, \alpha)$, which leads to $(1.7)$.

\section{REFERENCES}

[1] Goodman, A. W., Univalent functions. Vol. II, Mariner Pub. Co., Inc., Tampa, Florida, 1983.

[2] Ignaciuk, S., Parol, M., Zeros of complex polynomials and Kaplan classes, Anal. Math. 46 (2020), 769-779.

[3] Jahangiri, M., A gap condition for the zeroes of certain polynomials in Kaplan classes $K(\alpha, \beta)$, Mathematika 34 (1987), 53-63.

[4] Kim, Y. J., Merkes, E. P., On certain convex sets in the space of locally schlicht functions, Trans. Amer. Math. Soc. 196 (1974), 217-224.

[5] Royster, W. C., On the univalence of a certain integral, Michigan Math. J. 12 (1965), 385-387.

[6] Ruscheweyh, S., Convolutions in Geometric Function Theory, Séminaire de Math. Sup. 83, Presses de l'Université de Montréal, Montréal, 1982.

[7] Sheil-Small, T., Complex Polynomials, Cambridge University Press, Cambridge, 2002. 
Szymon Ignaciuk

Department of Applied Mathematics and Computer Science

University of Life Sciences in Lublin

ul. Głęboka 28

20-612 Lublin

Poland

e-mail: szymon.ignaciuk@up.lublin.pl

Maciej Parol

Department of Mathematical Analysis

The John Paul II Catholic University of Lublin

ul. Konstantynów $1 \mathrm{H}$

20-708 Lublin

Poland

e-mail: mparol@kul.lublin.pl

Received July 10, 2020 